\title{
Demonstrating hArtes project approach through an Advanced Car Information System
}

\author{
Francesco Piazza \\ DEIT - Università Politecnica \\ delle Marche \\ Via Brecce Bianche 1 \\ 60100 Ancona Italy \\ f.piazza@univpm.it \\ Paolo Peretti \\ DEIT - Università Politecnica \\ delle Marche \\ Via Brecce Bianche 1 \\ 60100 Ancona Italy \\ p.peretti@univpm.it
}

\author{
Stefania Cecchi \\ DEIT - Università Politecnica \\ delle Marche \\ Via Brecce Bianche 1 \\ 60100 Ancona Italy \\ s.cecchi@univpm.it \\ Ferruccio Bettarelli \\ Leaff Engineering \\ Via Puccini 75 \\ 62016 Porto Potenza Picena \\ (MC) Italy \\ bettarelli@leaff.it
}

\author{
Lorenzo Palestini \\ DEIT - Università Politecnica \\ delle Marche \\ Via Brecce Bianche 1 \\ 60100 Ancona Italy \\ I.palestini@univpm.it \\ Ariano Lattanzi \\ Leaff Engineering \\ Via Puccini 75 \\ 62016 Porto Potenza Picena \\ (MC) Italy \\ lattanzi@leaff.it
}

\begin{abstract}
The need for an advanced car information system has been recently emerging, able to handle issues left open by car information systems already on the market, and to overcome their limitations. In the hArtes framework an innovative approach is proposed, based on a general purpose system with support for human interfaces, an advanced remote application control (RAC) interface following a client-server paradigm, and (sub)systems implementing specific demanding functions. In this context a complex demonstrator, composed by 4 physical systems whose interfacing software is derived from $\mathrm{Nu}$-Tech DSP platform, is presented able to run computational-demanding CIS applications. The proposed architecture shows several advantages, such as increased flexibility, ease of integration with personal devices, software and hardware (individually replaceable modules) upgradeability.
\end{abstract}

\section{Keywords}

Advanced Car Information System, In Car audio system, Intelligent Transport Systems

\section{INTRODUCTION}

In the last years, pervasive information and communication technologies have driven a growing interest on the so-called Intelligent Transport Systems (ITS) whose major representative member is obviously the "Intelligent" Car. In fact, due to traffic congestion and growing distance from home to workplace, people spend more and more time in car, that hence becomes an appealing place to do many common activities such as listening to music and news, phone calling,

and doing many typical office tasks.

Therefore the car market is particularly sensible to the introduction of innovative services for drivers and passengers, also when security and driving aids are involved: Car Information Systems (CIS) is becoming a rapidly growing field of development [7, 19, 16].

Narrowing our focus to audio, the importance of in-car audio within the in-car Infotainment field has to be strongly remarked: the car is nowadays the most used audio listening environment indeed. The key role of in-car audio processing for infotainment purposes is justified by the fact that it lets the driver to concentrate on road and at the same time to manage many different functions in the CIS, like music, noise reduction, hands-free communication, automatic speech recognition, etc. Moreover, from the signal processing point of view, the wide band nature of the audio signal adds complexity while the requested quality calls for high precision signal processing, making in-car audio processing a very open research and development field. Although CIS systems are already on the market, many issues and trends are already open in the field. Therefore new architectures are needed to overcome the nowadays limitations.

In the hArtes European project (see later), an in-car demonstrator has been developed as a CIS system. The proposed architecture is termed Advanced CIS (ACIS) and it is composed by a management system, that is, a general purpose system with support for human interface and advanced Remote Application Control (RAC) and some embedded (sub)systems implementing specific signal processing demanding functions, following a client-server paradigm. Such architecture can face some issues and limitations of nowadays CIS. Its flexibility allows designing a wide variety of systems ranging from basic to high-end.

In Section 2, the aim of the hArtes project will be briefly recalled. In Section 3 the advanced car information system will be described in terms of both functionalities and architecture.

\section{HARTES PROJECT}


hArtes (Holistic Approach to Reconfigurable real Time Embedded Systems) is a three year (2006-2009) research project (Integrated Project) funded by the IST programme of the European Commission under the embedded systems action line. It aims to lay the foundation for a new holistic (endto-end) approach for complex real-time embedded system design, with the latest algorithm exploration tools and reconfigurable hardware technologies. The proposed approach will address, for the first time, optimal and rapid design of embedded systems from high-level descriptions, targeting a combination of embedded processors, digital signal processing and reconfigurable hardware. Therefore modular and scalable hardware platforms that can be reused and re-targeted by the tool chain to produce optimized realtime embedded products will be pursued. The results will be evaluated using advanced audio and video systems that support next-generation communication and entertainment facilities, such as immersive audio and in-car infotainment systems. Innovations of hArtes approach include: (a) support for both diagrammatic and textual formats in algorithm description and exploration, (b) a framework that allows novel algorithms for design space exploration, which aims to automate design partitioning, task transformation, choice of data representation, and metric evaluation for both hardware and software components, (c) a system synthesis tool producing near-optimal implementations that best exploits the capability of each type of processing element; for instance, dynamic reconfigurability of hardware can be exploited to support function upgrade or adaptation to operating conditions. From the application point of view, the complexity of future multimedia devices is becoming too big to design monolithic processing platforms. This is where the hArtes approach with reconfigurable heterogeneous system becomes vital. In the project, three different application scenarios in the multimedia area are considered: Immersive Audio Systems, Video Transcoders and in-vehicle CIS. In the following the CIS demonstrator is described, whose objectives are:

1. Develop an HW-SW system which effectively demonstrates the capability of the hArtes toolchain on a powerful heterogeneous reconfigurable hardware platform designed for the hArtes project. A high-end advanced CIS mounted in a real car constitutes a true real-life environment with great market potential.

2. Develop an innovative CIS system based on the hARTES technologies which also demonstrate the feasibility of the proposed approach.

3. Build a "car lab", i.e. create an overall system based on hArtes technologies that can be used as a strong dissemination tool, allowing interested groups to experiment new algorithms (i.e. test the efficiency of the hArtes toolchain) in a real ACIS framework, also remotely (see later).

\section{HARTES CIS}

Limitations of current CIS systems and the technological issues and trends strongly motivate the need of alternative CIS architectures incorporating the advantages of existing closed and open CIS solutions and able to successfully tackling the market requirements.

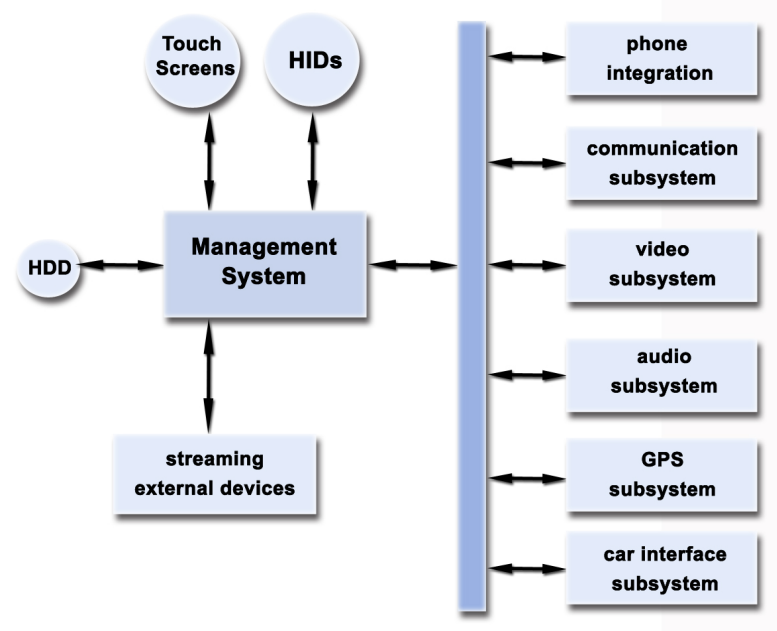

Figure 1: ACIS conceptual architecture

\subsection{The ACIS approach}

The proposed architecture is termed Advanced CIS (ACIS) and it is depicted in Fig. 1. Conceptually it is a combination of an Open System with general purpose CPU and well-known operating system for implementing interface and non-computational-intensive services (e.g. human interface devices, office, Web 2.0 services) and some specialized hardware subsystems for implementing computational intensive services (e.g. multimedia) connected through a network. More in detail, such architecture will comprise:

- a master system composed by a general purpose CPU running an open and stable operating system with support for human interface and advanced RAC following a client-server paradigm (with optional haptic devices);

- one or more optional slave systems, similar to the master system but with limited capabilities with respect to it;

- an optional interface with other car services and buses (e.g. CAN);

- one or more embedded (sub)systems implementing specific demanding functions such as audio processing, video processing, hands-free phone, etc. Such systems will offer the hardware and software needed to implement specific applications (e.g. audio player) with remote interface;

- an internal high speed network connecting all the systems;

- an always-on connection to the Internet.

In order to understand the functionality of the system, two different examples are briefly described:

1. Example A: High-end ACIS 
In this case the architecture is composed of four different physical systems connected by the internal network: one master installed in the dashboard, two slaves installed in the rear seats and an auxiliary system (called Power Server in the following) installed in the trunk.

The master (and partially the slaves) system interfaces with the user through some devices (touchscreen, haptic devices, voice, etc.), and with the car through some specific buses (e.g. CAN), controls the execution of remote computational demanding applications in the Power Server and allows to run seamlessly rich internet applications locally or remotely through the internet (Office, mail, etc.). The Power Server is able to run computational-demanding applications (especially multimedia) using specialized hardware and software (e.g. professional audio channels and algorithms, media storage, digital radio decoding) offering remote control through a client-server RAC model.

\section{Example B: Basic ACIS}

In this case the architecture is composed of only one physical system installed in the dashboard. The system offers all the capability of the master in the example A, together with some limited audio/video capabilities (e.g. four audio channels, DVD only storage), possibly supported by some specialized hardware (digital signal processor). However the client-server RAC model is maintained also in this case (e.g. using Loopback interfaces).

Through the uniform RAC interface, the proposed architecture allows overcoming the issues and limitations of nowadays CIS:

1. Different lifetime between vehicle and ACIS This problem is mainly related to the lifetime of the in-cabin systems, since their substitution requires additional interfacing/cabling, dismounting (sometimes dismantling) and remounting of cabin components (especially dashboard) with the consequent loss of the original car maker interior design. In the ACIS view, the master and slaves systems offer mainly human, car and application interfaces through easily upgradeable paradigms. Due to the interface nature of the systems the performance run is reduced allowing them to last likely for the whole car lifetime. Power Server type appliances can instead be easily upgraded.

2. Seamless integration of ACIS with consumer electronic personal devices The client-server rich internet applications paradigm can be effectively used to interface smart personal devices.

3. New telematics services for ACIS

Limiting the scope to infotainment services (excluding mission-critical ones), the always-on Internet connection is the most useful option, allowing to seamlessly integrate nowadays Web 2.0 applications and to stimulate the introduction of future car-specific ones (also based on the Semantic Web paradigms).

\section{Increasing performance}

The specialized hardware subsystems offer high-end performance, increased stability and flawless multimedia services. When a clear programming interface is defined, they allow also software upgradeability (algorithms and services) through a plug-in paradigm.

5. Stability and integration

Master (slave) system offers the capability to integrate car functionalities and support complex (multimedia) applications without loosing its stability. In fact either it simply runs the interface of remote applications in a sandbox (example A) or it runs the interface and some nonupgradable (well-written) simple audio/video applications (example B). In the example A the possible instability of the Power Server due to a badly written third-part application does not propagate to the master (slave).

6. Upgradeability and Aftermarket Compatibility The client-server RAC paradigm allows overcoming this problem. In fact substituting (example A) or adding (example B) the Power Server in the trunk allows upgrading the performance of the system (in term of functions, quality, speed, etc.) without any costly operation in the cabin. When automatic network configuration and service discovery mechanisms are implemented (e.g. ZeroConf, Bonjour, etc.) the upgrade process can be as simple as connecting the cables.

An ACIS system thus allows providing a variety of new and legacy functionalities: all the CIS services offered today plus some others and the capability of supporting new ones, as follows

- interface (human interface devices, application interfaces, etc.);

- personal devices integration;

- audio functions (playing, music archive, audio processing, etc.);

- video functions (playing, video archive, etc.);

- hands-free communication functions (extra/intra cabin, etc.);

- speech related functions (automatic speech recognition, speaker verification, etc.);

- communication and network functions;

- radio functions (analog, digital, satellite).

Navigation functions are not implemented since they are not of interest for the hArtes demonstration. Instead a full bunch of multimedia algorithms (especially audio) have been implemented to demonstrate the capability of the hArtes toolchain:

\section{- Algorithms for Enhanced In-Car Listening} development of scalable (microphones/loudspeakers) algorithms for audio quality enhancement, static and adaptive digital audio equalization, audio rendering. 


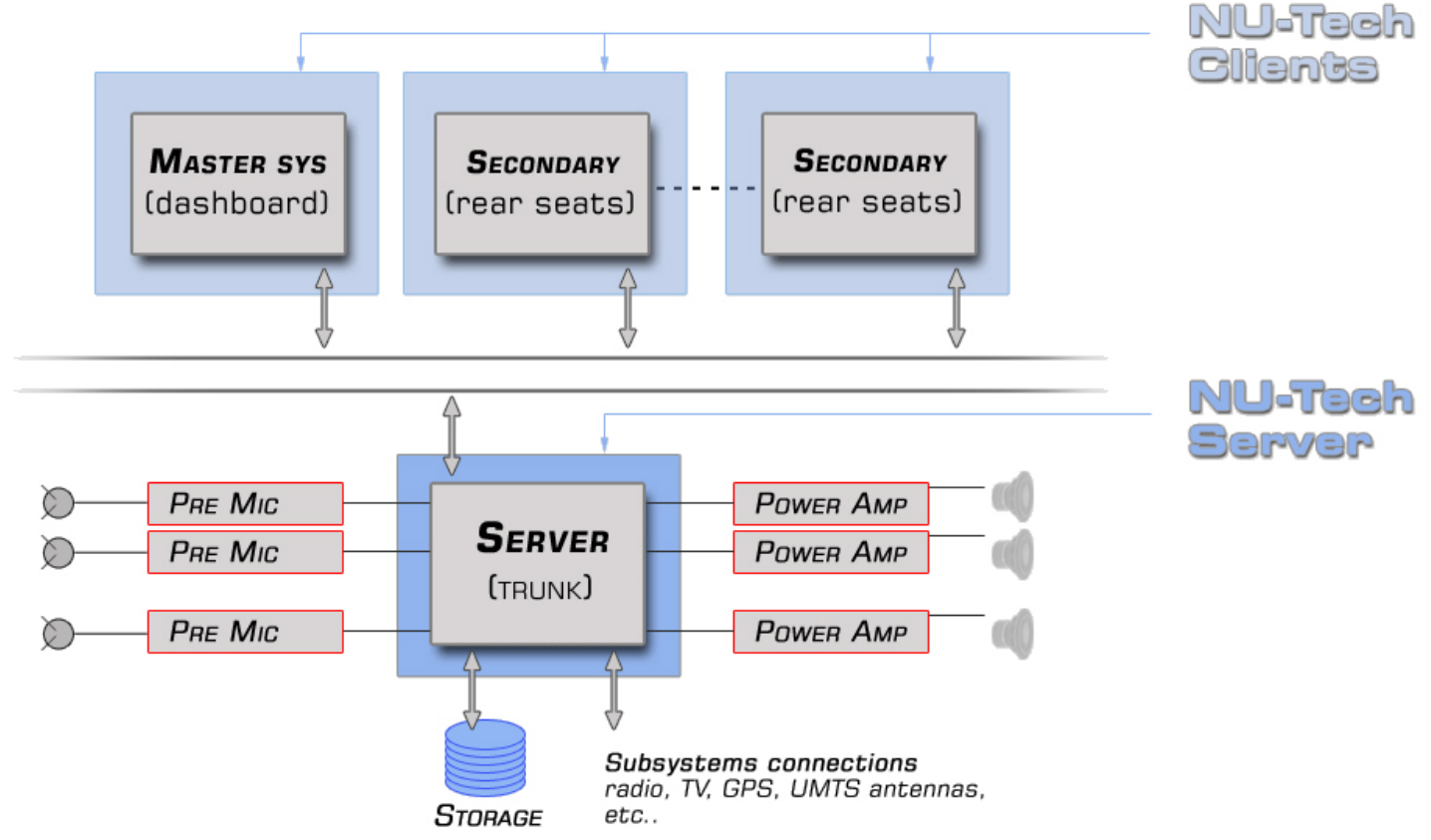

Figure 2: In Car demonstrator architecture

- Algorithms for Advanced In-Car Audio Communication

development of scalable (microphones/loudspeakers) algorithms for multichannel extra-cabin and intra-cabin communications, integrating acoustic echo cancellation and noise reduction strategies.

- Algorithms for Automatic Speech and Speaker Recognition

improved man-machine interface for command and control of software applications through automatic speech recognition, speaker verification technology for secured access to in-car services.

- Algorithms for video algorithms for the coding/decoding and distribution of video contents to the systems inside the cabin.

- Algorithms for digital radio receiver algorithms for the decoding of the Digital Radio Mondiale signal as proof of concept of the capability of implementing soft radio.

Since the demonstrator has to be shared among a group of geographically distributed partners and also in order to help dissemination using Internet, an additional service has been implemented called Network Remote Control (NRC). This service will allow a remote control of the upload and execution of selected audio algorithms on the ACIS, selectively recording the acoustic events inside the cabin for successive downloading and analysis.

\subsection{System architecture}

The demonstrator will be therefore composed of the following physical systems embedded in the selected vehicle (Fig. 2):

\section{- Master system}

The Master system will be mounted in the car dashboard providing at least the following hardware functionalities:

- user interface and visualization means such as a high resolution display;

- HID such as touch screen, buttons, wheels, removable keyboard, etc;

- a (stereo) audio channel for headphone private listening;

- USB 2.0 interfaces for user and Personal Devices;

- network connection.

Such a system will be an Open System, hence implemented as a Carputer (Car PC) driven by a stable Operating System. Specific software will be available for the user interface and the interface with the Power Server, based on a client-server RAC model with a proper resource declaration mechanism. Interfaces to lower layers (e.g. CAN) can be considered.

- Slave system(s) One (or more) system will be mounted in the car rear seats providing the same functionalities of the master system, but with a limited control over the system resources.

- Power Server

The Power Server system will be mounted in the car trunk providing at least the following hardware functionalities: 
- media (audio, video) storage;

- audio (possibly video) digital high quality input/ output channels;

- adequate audio/video processing capability;

- GSM support for phone calling/answering;

- hardware support for radio/TV tuners;

- hardware support for GPS;

- network interface.

Such an embedded system will be based on the hArtes hardware platform and will be able to run executable code generated by the hArtes toolchain. Moreover, possibly inside an embedded operating system, specific software will be available for loading code on the platform, controlling the execution and implementing the application interface with the Master/Slave systems, based on a client-server RAC model. The system also will implement the Network Remote Control functionality when connected to the network, i.e. authenticated users can remotely (and securely) upload algorithm code and execute it, setting the number and position of microphones/loudspeakers and recording the audio results in the cabin (with microphones and/or a mannequin). A two step strategy has been chosen to develop the Power Server and to reduce risks and efforts. The first step is to develop a Carputer-based prototype to test and validate the multichannel audio system and the algorithms inside the car, before the availability of the final hArtes platform. Once the hArtes hardware platform will be available it will be integrated with the already developed system.

- Internet Connection The always-on connection to the Internet will be realized using a UMTS High-Speed Downlink Packet Access channel or a downgraded compatible channel (GPRS) where UMTS is unavailable. In fact although specific Vehicle to Vehicle - Vehicle to Infrastructure (V2V-V2I) communication proposals are emerging, see for example [6], the UMTS/GPRS channel is today (and in the near future) the only widely available Internet connection mean for vehicles through every EU countries, see for example [1].

- Vehicle Network A suitable network connection is required to connect the systems together. Such a network is the top layer of the vehicle network architecture. In the automotive infotainment, MOST (Media Oriented Systems Transport) has emerged as a standard [12] and therefore must be considered as a candidate. IP-based Ethernet networking is also attracting interest and has been already used in the infotainment field and selected for the hArtes demonstrator; for a vehicle network review see [11].

\subsection{NU-Tech based RAC Interface}

The innovative RAC interface is needed for running the developed algorithms, interfacing them to the available hardware and integrating together. The developed framework is based on the Nu-Tech DSP platform [10, 18]. NU-Tech is a powerful DSP platform to validate and real-time debug complex algorithms, simply relying on a common PC. An easy NU-Module Schema plug-in architecture and a free SDK let

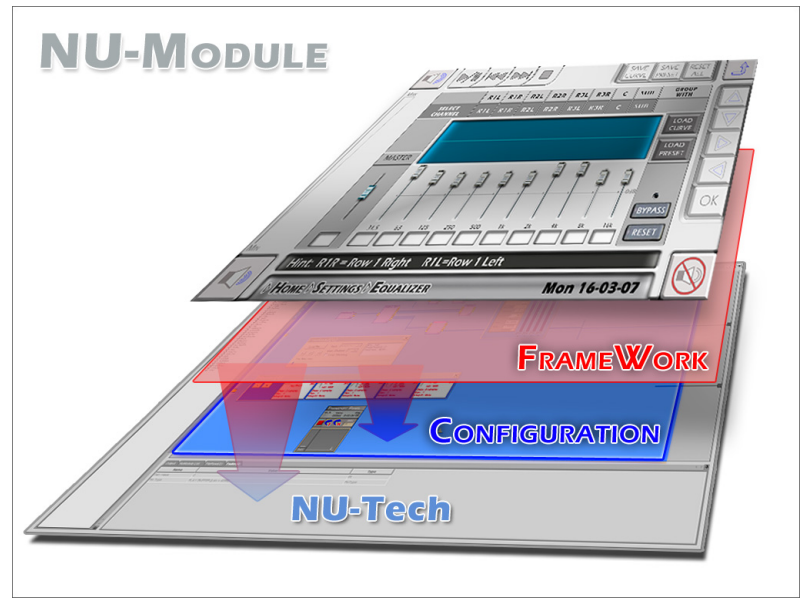

Figure 3: NU-module schema

the developer write his own NU-Tech Satellites (NUTSs) in $\mathrm{C}++$ and immediately plug them into the graphical interface design environment. A low-level ASIO 2.1 interface allows minimum and repeatable latencies, fully exploiting soundcard hardware resources. Besides a proprietary algorithm allows full synchronization between audio/video streams on different systems over a network. Moreover recently introduced NU-Tech modules (NU-Modules) technology let the design of brand new applications to be more easy and straightforward: a module is a configuration of NUTSs managed by a high level logical layer which also handles all the NU-Tech actions (starting streaming, making connections, etc ). A module can have its own GUI and can control NUTSs parameters, NUTSs interconnections, simple and complex procedures (i.e. car acoustic ambient characterization through different identification techniques)(Fig. 6). NU-Tech helps the developer to describe an application and support algorithm exploration and validation through simulation and graphical visualization. In the hArtes platform, NU-Tech Kernel has been rewritten using exclusively Win32 features, an enhanced Audio Streaming engine which supports both DirectSound and ASIO 2.1 drivers has been designed. An enhanced Video Streaming support has been also introduced with an Audio/Video synchronization mechanism which allows the streaming to be slaved to external signal generators (audio, video etc., time code). The algorithms are integrated as one or more NUTS (plug-in) whose behaviour can be remotely controlled at runtime using Client/Server architecture. A NU-Module will be able to control NUTSs placed in different NU-Tech installed in different PCs over an IP network (Fig. 3). In-Car video streaming can be taken as an example: the audio is played through the audio system while the video is processed by the Power Server and streamed to and played by the Master (Slave) system in the cabin (without loosing synchronization) which in turn controls its playback.

\section{ACIS DEMONSTRATOR}

The selected vehicle for the demonstrator is a Mercedes R320

CDi V6 Sport car, which has been chosen because of:

- Space (needed by the Carputer hardware and audio 
systems)

- 3 rows of seats (needed by some algorithms)

- Second row with 2 seats (easier implementation of rear systems, no central seat to be considered by the algorithms)

- Easy integration of part of the system in the dashboard

- New and prestigious model, useful for dissemination

The car has been then equipped with up to 30 loudspeakers (ad-hoc designed by one of the hArtes partners) and 24 microphones located inside the car according to algorithms development requirements. The ACIS system has to control each single loudspeaker channel for implementing high-end audio/video algorithms. Therefore every single loudspeaker has a dedicated high quality automotive power amplifier. Professional ASIO soundcards have been used to manage all the system I/Os. It's out of the scope of this paper to describe the system architecture into deep. It will be object of further works already under development. The system has been thought on a client-server architecture as shown in Fig. 4. The PowerServer is now a PC able to run computational demanding applications and manage all the I/O through soundcards where amplifiers/loudspeakers and microphones are connected. A number of different clients can then connect over a LAN with the PowerServer and interact with it. Clients are located one in the car dashboard and one per each rear row. A NU-Tech based application runs on each client as a shell presenting the user with a simple Graphical User Interface (GUI) letting him interact with the whole system. Typical multimedia functionalities have been implemented such as: audio playback, video playback, playlist handling, phone communication; together with more sophisticated functionalities: complex audio processing for hands-free phone communication, speech reinforcement, automatic speech and speaker recognition. The user is also able to configure the system according to his particular preference thanks to custom GUIs implementing crossover, channel mapping, equalization features and so on.

\section{ALGORITHMS IMPLEMENTATION AS NUTS}

As already said, the algorithms are integrated as one or more NUTS (plug-in) whose behaviour can be remotely controlled at run-time using Client/Server architecture. To ease the developer in quickly creating new NUTSs without having to deal with GUI programming, NU-Tech provides a built-in window called "RealTime Watch" (RTWatch) to be associated to each NUTS as depicted in Fig. 5. The developer can choose, by code, to expose some NUTS' internal variables on this window, and effectively control his plug-in [9]. This feature has been used in hArtes algorithm implementation as demonstrated by the following examples: a digital crossover, a parametric equalizer, a graphic equalizer and a fractional delay $[3,14]$.

\subsection{Digital Crossover}

High quality loudspeakers used in nowadays audio systems can be roughly classified depending on their different emission frequency bands: sub-woofer (for frequencies lower than

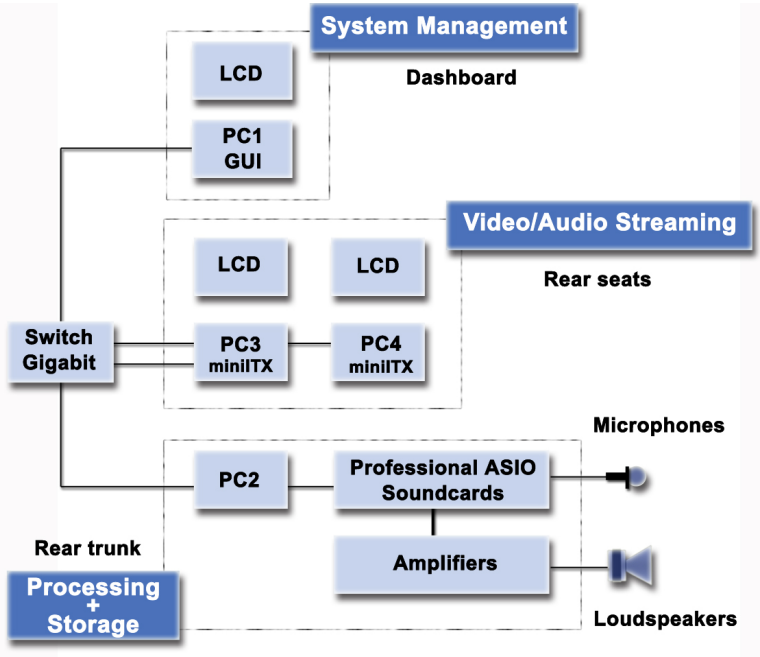

Figure 4: PC Only ACIS demonstrator

$100 \mathrm{~Hz}$ ), woofer (between $100 \mathrm{~Hz}$ and $300 \mathrm{~Hz}$ ), mid-range $(300 \mathrm{~Hz}-3 \mathrm{kHz})$ and tweeter $(3 \mathrm{kHz}-20 \mathrm{kHz})$. In order to avoid distortions and drivers damages it is necessary to develop a crossover network able to split the audio spectrum into desired bands. A FIR tree structure ensuring linear phase and low computational cost due to fast FIR filtering implemented in the frequency domain $[20,2,4]$ has been implemented through the NU-Tech Platform. From the RTWatch tab the user is allowed to change the number of output channels and filters cut-off frequency. For filter design, the transition bandwidth and the order are estimated from the cut-off frequency and the sample rate through an empirically obtained relationship. The chosen transition bandwidths properly decrease with decreasing frequencies, filters orders are calculated in order to ensure at least $60 \mathrm{~dB}$ attenuation in the stop band.

\subsection{Parametric Equalizer}

As pointed out in [8], car is a very complex acoustic environment when compared to a normal size listening room. Car cabin frequency response is strictly influenced by the position of loudspeakers and the shape of the car cabin; the reflected sounds can attenuate or amplify the direct sound from the loudspeakers introducing irregularities in the frequency response. A parametric equalizer is a useful tool in order to correct frequency responses and also to amplify low frequency components masked by the background noise. A NUTS has been realized starting from the high order Butterworth and Chebyshev filter design technique presented in [13], obtaining sharper frequency responses, with respect to the conventional biquadratic designs. The input signal is filtered by up to five cascading parametric equalizers, depending on the "Enabled" control state of each filter provided by RTWatch tab. For each filter, it is possible to change the Filter type (Butterworth or Chebychev I and II type), the design parameters (bandwidth and cut gains, cut-off frequencies and bandwidths), and the filter Order, even during streaming. Upon change of Sample Rate, filters are automatically redesigned. A Bypass control allows to make the equalizer transparent at any moment. 


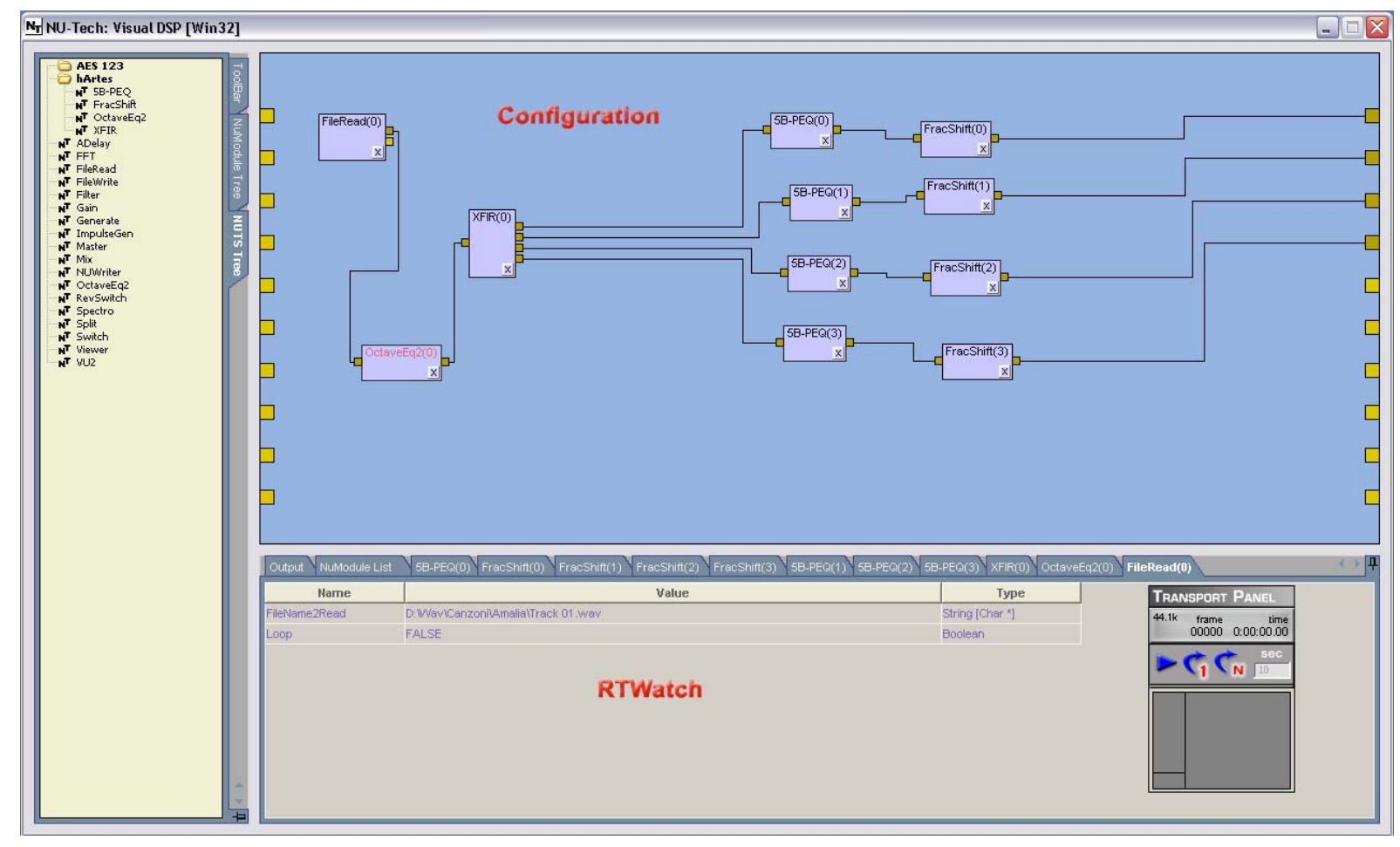

Figure 5: An Example of NU-Tech development board

\subsection{Graphic Equalizer}

Equalization is implemented to enhance tone quality and modify frequency response. Equalizers are used to compensate for speaker placement, listening room characteristics (e.g., in automotive application, to have low frequencies emphasized in the presence of background noise), and to tailor to personal taste (e.g. to enhance the listening experience of particular kinds of music). This compensation is accomplished by cutting or boosting, that is, attenuating or amplifying a range of frequencies. There are two well known paradigms for digital audio equalization: the parametric and the graphic approaches. A graphic equalizer is a high-fidelity audio control that allows the user to see graphically and control individually a number of different frequency bands for each channel. The solution adopted is based on an FFT approach ensuring linear phase and low computational cost due to fast FIR filtering implemented in the frequency domain $[17,5]$. An octave band EQ NUTS has been realized: from the RTWatch tab the user is allowed to change the gain of each band and flatten the equalization curve through the "Flat" button. The equalizer has been implemented by the Weighted Overlap and Add (WOLA) method [15] with a FFT size of 4096 and a decimation factor of 4 . If the Sample Rate changes, the equalization filter is recalculated in order to retain the current normalized equalization curve. For low sample rates, bands whose lower cut-off frequency is higher than Nyquist frequency are obviously non influential.

\subsection{Fractional Delay}

In a vehicle cabin the loudspeaker location possibilities are rather restricted. Off-center listening position is inevitable. The delay difference should be compensated through digital delay to equalize the sound arrival times from loudspeakers to the listening position. In this context a fractional delay can be used to achieve a better sound alignment. Fractional delay can also be useful for the task of adjusting the phase of signals, and aligning different audio streams. Among the various approaches reported in literature, we chose to resort to a computationally efficient variable fractional delay approach, based on coefficients polynomial fitting [21]. A FracShift NUTS has been realized as a standard C ++ dll file able to operate within the NU-Tech interface. From the RTWatch tab the user is allowed to change the value of decimal value of the delay (between 0 and 100, where 100 represents an integer delay of one sample), also during streaming. The filtering process inevitably introduces an integer delay of 50 samples: the overall delay is displayed on the output tab.

\section{CONCLUSIONS}

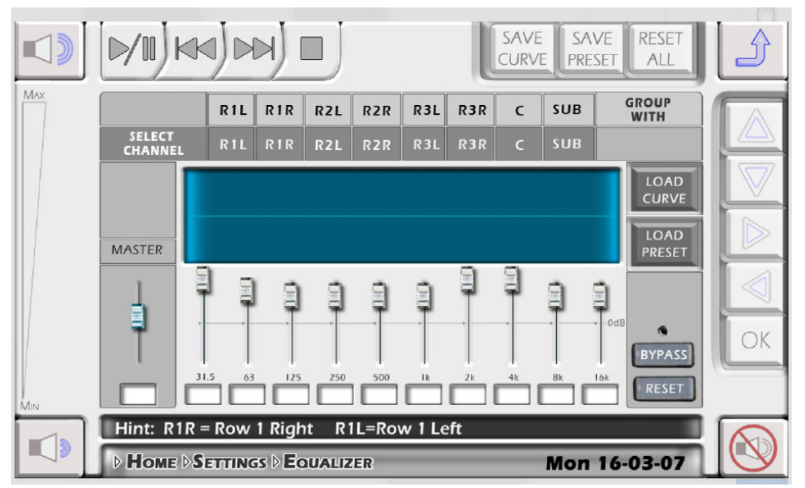

Figure 6: Equalizer NU-module GUI 
The hArtes project has been introduced and the ACIS demonstrator has been presented as a proof-of-concept. The main functionality and the architecture of the overall system have been illustrated highlighting all the advantages of this approach. NU-Tech description has been reported and its role as framework of the system presented. NU-Tech approach to algorithms design has been then shown through some ACIS algorithms examples. In the next future the on going work of the project will be further presented to scientific community and results presented through new papers.

\section{ACKNOWLEDGMENTS}

This work was supported by the European Commission as sponsor of the hArtesProject number 035143.

\section{ADDITIONAL AUTHORS}

Additional authors:

Emanuele Moretti (DEIT - Università Politecnica delle Marche, email: emanuele.moretti@univpm.it) and Emanuele Ciavattini (Leaff Engineering, email: ciavattini@leaff.it)

\section{REFERENCES}

[1] eCall. http://www.esafetysupport.org/en/ecall_toolbox/, 2007.

[2] S. Azizi, H. Hetzel, and H. Schöpp. Design and implementation of linear phase crossover filters using the FFT. 98th AES Convention, Proceedings of, January 1995.

[3] S. Cecchi, P. Peretti, L. Palestini, F. Piazza, F. Bettarelli, and A. Lattanzi. Real time implementation of an innovative digital audio equalizer. 123th AES Convention, Proceedings of, october 2007.

[4] I. M. Dukic, D. Z. Todorovic, and L. D. Milic. The influence of impulse response length and transition bandwidth of magnitude complementary crossover on perceived sound quality. 120th AES Convention, Proceedings of, May 2006.

[5] A. J. S. Ferreira and J. M. N. Vieira. An efficient 20-band digital audio equalizer. 98th AES Convention, Proceedings of, January 1995.

[6] GST. Global system for telematics. http://www.gstforum.org/, 2006.

[7] R. K. Jurgen. Automotive software. SAE Ed., 2006.

[8] J. Kontro, A. Koski, J. Sjoberg, and M. Vaananen. Digital car audio system. IEEE Transactions on Consumer Electronics, 39(3), August 1993.

[9] A. Lattanzi, F. Bettarelli, and S. Cecchi. NU-Tech: the entry tool of the hArtes toolchain for algorithms design. accepted at 124th AES Convention, June 2008.

[10] Leaff Engineering. Nu-tech dsp platform. http://www.nu-tech-dsp.com/, 2007.

[11] C. A. Lupini. Multiplex bus progression. SAE 2003 World Congress and Exhibition, Proceedings of, March 2003.

[12] MOST. Media oriented systems transport. http://www.mostnet.de, 2006.

[13] J. S. Orfanidis. High-order digital parametric equalizer design. J. Audio Eng. Society, 53(11), November 2005.
[14] L. Palestini, P. Peretti, S. Cecchi, F. Piazza, A. Lattanzi, and F. Bettarelli. Linear Phase Mixed FIR/IIR Crossover Networks: Design and Real-Time Implementation. 123th AES Convention, Proceedings of, october 2007.

[15] L. R. Rabiner and R. E. Crochiere. Prentice-Hall, Inc., 1996.

[16] SAE, editor. SAE International Conference "Convergence 2006", 2006.

[17] H. Schopp and H. Hetzel. A linear phase 512 band graphic equalizer using the fast fourier transform. 96th AES Convention, Proceedings of, January 1994.

[18] S. Squartini, E. Ciavattini, A. Lattanzi, D. Zallocco, F. Bettarelli, and F. Piazza. NUTech: implementing DSP algorithms in a plugin based software platform for real time audio applications. 118th AES Convention, Proceedings of.

[19] L. Vlacic, M. Parent, and F. Harashima. Intelligent vehicle technologies. SAE Ed., 2001.

[20] R. Wilson, G. Adams, and J. Scott. Application of digital filters to loudspeaker crossover networks. $J$. Audio Eng. Society, 37(6):445-464, June 1989.

[21] H. Zhao and J. Yu. A simple and efficient design of variable fractional delay FIR filters. Circuits and Systems II: express briefs, IEEE transaction on, 53(2), February 2006. 